\title{
Effects of high-quality nursing on puerpera with pregnancy hypertension.
}

\author{
Fei Li ${ }^{*}$
}

Department of Obstetrics, Zhuji Maternal and Child Care Service Center, Zhuji 311800, Zhejiang, PR China

\begin{abstract}
Objective: This paper aims to discuss the clinical effects of high-quality nursing for puerpera with pregnancy hypertension.

Methods: A total of 88 puerpera cases with pregnancy hypertension were collected from March 2016 to July 2017. Cases were randomly divided into two groups: control group $(n=44)$ and intervention group $(n=44)$. The control group was provided with conventional nursing, whereas the intervention group was treated with high-quality nursing. Comparisons were made on psychological state, blood pressure levels, incidences of pre-eclampsia and eclampsia, nursing satisfaction, newborn birth weight, and Apgar scores of the two groups.

Results: Depression and anxiety scores of intervention group are significantly lower than those of control group $(\mathbf{P}<\mathbf{0 . 0 5})$. Diastolic pressure level and systolic pressure level of intervention group are far lower than those of the control group $(\mathbf{P}<\mathbf{0 . 0 5})$. Incidences of pre-eclampsia and eclampsia in the intervention group reached $18.2 \%$ and $4.5 \%$, respectively, which are significantly lower than those of the control group (38.6\% and $20.5 \%)(\mathrm{P}<0.05)$. Nursing satisfaction of intervention group total of $97.7 \%$, which is significantly higher than that of the control group $(81.8 \%)(P<0.05)$. Newborn birth weight and Apgar scores of intervention group are significantly higher than those of control group $(\mathbf{P}<0.05)$.

Conclusions: High-quality nursing features outstanding clinical effect on puerpera with pregnancy hypertension, improves psychological state and blood pressure level, and increases newborn birth weight effectively. Hence, high-quality nursing is a characteristic of safety and reliability and worthy of clinical promotion and applications.
\end{abstract}

$\underline{\text { Keywords: Pregnancy hypertension, High-quality nursing, Psychological state, Blood pressure level. }}$

Accepted on November 25, 2017

\section{Introduction}

Pregnancy hypertension is a common obstetrical disease. The incidence of pregnancy hypertension increases year by year [1]. This condition is caused by full-body arteriospasm and will induce hypertension and proteinuria of puerpera. Delayed treatment may cause catastrophic consequences to puerpera or death [2]. Therefore, puerpera with pregnancy hypertension must be prevented and diagnosed. Meanwhile, proper nursing shall be provided to protect health and safety of puerpera and newborns [3]. In this paper, 88 puerpera cases with pregnancy hypertension were collected from March 2016 to July 2017 to discuss clinical effects of high-quality nursing.

\section{Information and Methods}

\section{General information}

In this study, 88 puerpera cases with pregnancy hypertension were collected in our hospital from March 2016 to July 2017 and randomly divided into two groups: control group $(n=44)$ and intervention group $(n=44)$. The following data were obtained for the control group: age of 22-41 years old, with an average of $30.1 \pm 2.8$ years; $30-40$ weeks of gestation, with average of $37.8 \pm 1.5$ weeks; included 20 cases of primipara and 24 cases of multipara. On the other hand, the following information were obtained for the intervention group: age of 22-41 years old, with average of $30.3 \pm 2.9$ years; at $30-39$ weeks of gestation, with an average of $37.1 \pm 1.4$ weeks; comprised 18 cases of primipara and 26 cases of multipara. General information of the two groups were processed by SPSS 22.0. No significant difference was observed $(\mathrm{P}>0.05)$.

\section{Methods}

Nursing method of control group: Control group was provided with conventional nursing. Dynamics of vital signs were observed, and medications were strictly guided according to doctors' advice. Puerpera were asked to consume light diet and were provided with position nursing.

Nursing method of the intervention group: Based on conventional nursing of the control group, the intervention group was treated with high-quality nursing. Detailed contents were as follows: (1) Psychological content: most puerpera with pregnancy hypertension are concerned with the disease and are afraid of influencing newborns; thus, they exhibit negative 
emotions such as anxiety and depression. Therefore, nurses actively communicated with puerpera, evaluated their psychological state, and comprehended their psychological appeals, thus enabling them to provide personalized psychological counseling and relieve negative emotions of puerpera. At the same time, nurses introduced knowledge of the disease to patients and imparted comprehensive health education to make patients recognize the disease accurately and face it positively. In addition, nurses introduced successful cases to enhance confidence of patients and protect health of both puerpera and newborns to the maximum extent. (2) Ward environment: ward was kept clean, neat, and hygienic all time. Temperature, humidity, and illumination were strictly controlled to promote good rest of puerpera. Patients were also asked to lie on bed and to obtain adequate sleep to accelerate recovery. All nursing acts were performed gently to avoid disturbance of patients' rest or cause other accidents. (3) Disease observation: blood pressure was tested every $2 \mathrm{~h}$ for patients with light hypertension (140-150/90-100 $\mathrm{mmHg}$ ) and every $30 \mathrm{~min}$ for patients with moderate hypertension (150-160/100-110 $\mathrm{mmHg}$ ). Electrocardiography monitoring was performed when necessary. At the same time, vital signs of puerpera and fetus conditions were observed dynamically. Nurses reported all abnormal conditions to the doctor and assistant doctors for timely and proper treatment. (4) Nursing for pre-eclampsia or eclampsia: pregnancy hypertension can induce pre-eclampsia and eclampsia, thus threatening safety and health of puerpera and newborns. Pregnancy hypertension is a primary cause of puerpera death. Hence, hierarchical nursing for pre-eclampsia or eclampsia was provided. At convulsion of puerpera, mouth gag was performed immediately to insert conduit for oxygen uptake, sputum suction, and smooth respiration. Additionally, medications to spasmolysis, decompression, and sedation and diuretics were administered to patients. (5) Nursing after delivery: after smooth delivery of puerpera, nurses enhanced ward inspection, regularly observed for uterine contraction, and evaluated vaginal bleeding of puerpera to prevent complications. Blood pressure level was also observed regularly to prevent eclampsia to the maximum extent.

Observation indexes: Psychological state, blood pressure level, incidences of pre-eclampsia and eclampsia, nursing satisfaction, newborn birth weight, and Apgar scores of the two groups were compared. (1) Psychological state: depression degree of puerpera was evaluated using self-rating depression scale with scores ranging from $0-100$. Critical score is at 50, light depression at 50-60, moderate depression at 61-70, and severe depression at $>70$. Higher scores indicate more severe depression. Conversely, anxiety degree of puerpera was evaluated using self-rating anxiety scale using scores of 0-100. Similarly, critical score is at 50, with light anxiety scored at 50-59, moderate anxiety at 61-70, and severe anxiety at $>70$. Higher scores indicate more severe anxiety. (2) Blood pressure indexes, including diastolic and systolic pressure, were also observed. (3) Nursing satisfaction: Nursing satisfaction of puerpera was evaluated by a questionnaire survey using scores of $0-100$, with nursing care considered very satisfying at $>90$, satisfying at $60-90$, and unsatisfying at scores $<60$. The sum of very satisfying and satisfying scores corresponds to nursing satisfaction. (4) Apgar scores of the newborn were rated with scores of $0-10$, with heavy asphyxia scored at $0-3$, light asphyxia at 4-7, and normal condition at scores $>7$.

\section{Statistical analysis}

Observation data of two groups were processed by SPSS 22.0. Measurement data were expressed as $( \pm s)$ and examined by $t-$ test. Enumeration data were expressed as percentage and examined by $\chi^{2}$-test, where $\mathrm{P}<0.05$ reflects significant difference between the two groups.

\section{Results}

\section{Psychological state}

Depression score and anxiety score of the intervention group are significantly lower than those of the control group $(\mathrm{P}<0.05)$ (Table 1)

Table 1. Psychological state of the two groups ( $x \pm s$, scores).

\begin{tabular}{lll}
\hline Groups & Depression score & Anxiety score \\
\hline Intervention group $(n=44)$ & $39.8 \pm 4.8$ & $38.7 \pm 4.6$ \\
\hline Control group $(n=44)$ & $44.5 \pm 5.3$ & $43.1 \pm 5.0$ \\
\hline$t$ & 4.3599 & 4.2958 \\
\hline$P$ & 0.0000 & 0.0000 \\
\hline
\end{tabular}

\section{Blood pressure level}

Diastolic pressure and systolic pressure of the intervention group are lower than those of the control group $(\mathrm{P}<0.05)$ (Table 2).

Table 2. Blood pressure level of the two groups $(x \pm s, \mathrm{mmHg})$.

\begin{tabular}{lll}
\hline Groups & Diastolic pressure & Systolic pressure \\
\hline Intervention group $(n=44)$ & $90.3 \pm 3.5$ & $129.9 \pm 5.6$ \\
\hline Control group $(n=44)$ & $100.2 \pm 4.6$ & $152.7 \pm 6.2$ \\
\hline$t$ & 11.3611 & 18.1022 \\
\hline$P$ & 0.0000 & 0.0000 \\
\hline
\end{tabular}

\section{Pre-eclampsia and eclampsia}

Incidences of pre-eclampsia and eclampsia in the intervention group reached $18.2 \%$ and $4.5 \%$, respectively, which are significantly lower than those of the control group $(38.6 \%$ and $20.5 \%)(\mathrm{P}<0.05)$ (Table 3).

Table 3. Pre-eclampsia and eclampsia of the two groups.

\begin{tabular}{lll}
\hline Groups & Pre-eclampsia & Eclampsia \\
\hline Intervention group $(n=44)$ & $8(18.2)$ & $2(4.5)$ \\
\hline
\end{tabular}




\begin{tabular}{lll}
\hline Control group $(\mathrm{n}=44)$ & $17(38.6)$ & $9(20.5)$ \\
\hline $\mathrm{X}^{2}$ & 4.5257 & 5.0909 \\
\hline $\mathrm{P}$ & 0.0333 & 0.0240 \\
\hline
\end{tabular}

\section{Nursing satisfaction}

Nursing satisfaction of the intervention group total of $97.7 \%$, which is significantly higher than that of the control group $(81.8 \%)(\mathrm{P}<0.05)$ (Table 4).

Table 4. Nursing satisfaction of the two groups.

\begin{tabular}{|c|c|c|c|c|}
\hline Groups & $\begin{array}{l}\text { Very } \\
\text { satisfying }\end{array}$ & $\begin{array}{l}\text { Satisfyi } \\
\text { ng }\end{array}$ & $\begin{array}{l}\text { Unsatisfyi } \\
\text { ng }\end{array}$ & $\begin{array}{l}\text { Nursing } \\
\text { satisfaction }\end{array}$ \\
\hline $\begin{array}{l}\text { Intervention group } \\
(\mathrm{n}=44)\end{array}$ & $33(75.0)$ & $10(22.7)$ & $1(2.3)$ & $43(97.7)$ \\
\hline Control group $(n=44)$ & $28(63.6)$ & $8(18.2)$ & $8(18.2)$ & $36(81.8)$ \\
\hline$x^{2}$ & & & & 6.0647 \\
\hline $\mathrm{P}$ & & & & 0.0137 \\
\hline
\end{tabular}

\section{Newborn weight and Apgar scores}

Newborn weight and Apgar scores of the intervention group are significantly higher than those of control group $(\mathrm{P}<0.05)$ (Table 5).

Table 5. Newborn weight and Apgar scores of the two groups $(x \pm s)$.

\begin{tabular}{lll}
\hline Groups & Weight $(\mathbf{k g})$ & Apgar (scores) \\
\hline Intervention group $(\mathrm{n}=44)$ & $3.4 \pm 0.4$ & $9.5 \pm 0.5$ \\
\hline Control group $(\mathrm{n}=44)$ & $2.4 \pm 0.5$ & $8.1 \pm 0.6$ \\
\hline $\mathrm{t}$ & 10.3593 & 11.8902 \\
\hline $\mathrm{P}$ & 0.0000 & 0.0000 \\
\hline
\end{tabular}

\section{Discussion}

Pregnancy hypertension is a unique hypertension disease that commonly occurs in obstetrics department. This condition is characterized by serious symptoms and high incidence [4]. Delayed treatment will cause pre-eclampsia, eclampsia, and placental abruption, thus influencing health of both puerpera and newborns. Furthermore, incidence of pregnancy hypertension increases year by year, whereas age of onset decreases gradually [5]. Delayed effective intervention not only threatens life and health of puerpera but also influences delivery outcomes.

High-quality nursing is a comprehensive nursing mode which provides assistance in developing confidence of patients to treatment, allow them to face the disease positively, and maintain the best psychological and physical states by proper intervention to control the disease, relieve symptoms, accelerate recovery, and achieve outstanding clinical effects [6,7]. High-quality nursing to puerpera with pregnancy hypertension mainly focuses on psychological nursing, ward environment nursing, disease observation, nursing for preeclampsia and eclampsia, and post-delivery nursing [8,9]. This type of care provides comprehensive, systematic, and thorough nursing services to puerpera, thus improving outcomes of puerpera and newborns [10].

Research results demonstrate that depression score, anxiety score, diastolic and systolic pressure levels, and incidences of pre-eclampsia and eclampsia in the intervention group are significantly lower than those of control group $(\mathrm{P}<0.05)$. The intervention group achieved significantly better nursing satisfaction $(\mathrm{P}<0.05)$ and featured significantly higher newborn weight and Apgar score compared with the control group $(\mathrm{P}<0.05)$. These index values are close to those found in related literature. Therefore, high-quality nursing can improve psychological state of puerpera, control blood pressure level, reduce complications, increase nursing satisfaction, accelerate recovery of puerpera, and improve newborn outcomes and features high clinical value.

\section{Conclusion}

To sum up, high-quality nursing for puerpera with pregnancy hypertension results in outstanding clinical effects. Not only can this type of care improve psychological state and blood pressure level of puerpera but also increase quality of newborns. High-quality nursing is a characteristic of safety and reliability and is worthy of clinical promotion and applications.

\section{References}

1. Roberts CL, Algert CS, Morris JM, Ford JB. Increased planned delivery contributes to declining rates of pregnancy hypertension in Australia: a population-based record linkage study. BMJ Open 2015; 5: e009313.

2. Preethi DMD, Jayanthi VE. SOM clustering approach: investigation on cataract surgery structural changes in iris. Biomed Res-India 2017; 28: 5210-5216.

3. Weissgerber TL, Turner ST, Mosley TH, Kardia SLR, Hanis CL, Milic NM, Garovic VD. Hypertension in Pregnancy and Future Cardiovascular Event Risk in Siblings. J Am Soc Nephrol 2016; 27 : 894-902.

4. Webster LM, Conti-Ramsden F, Seed PT, Webb AJ, Nelson-Piercy C, Chappell LC. Impact of antihypertensive treatment on maternal and perinatal outcomes in pregnancy complicated by chronic hypertension: A systematic review and meta-analysis. J Am Heart Assoc 2017; 6: e005526.

5. Goyal K, Agarwal R. Pulse based sensor design for wrist pulse signal analysis and health diagnosis. Biomed ResIndia 2017; 28: 5187-5195.

6. Mustafa R, Ahmed S, Gupta A, Venuto RC. A Comprehensive Review of Hypertension in Pregnancy. J Pregnancy 2012; 2012: 105918.

7. Campbell SK, Lynch J, Esterman A, McDermott R. Prepregnancy predictors of hypertension in pregnancy among Aboriginal and Torres Strait Islander women in north Queensland, Australia; a prospective cohort study. BMC Public Health 2013; 13: 138. 
8. Arain MI, Ghoto MA, Dayo A. Evaluation of awareness regarding hypertension and their therapy among hypertensive patients at hospital of Hyderabad, Pakistan. Lat Am J Pharm 2017; 36: 1942-1946.

9. Mehta B, Kumar V, Chawla S, Sachdeva S, Mahopatra D. Hypertension in pregnancy: A community-based study. Indian J Community Med 2015; 40: 273-278.

10. Barron C, Mandala M, Osol G. Effects of pregnancy, hypertension and nitric oxide inhibition on rat uterine artery myogenic reactivity. J Vasc Res 2010; 47: 463-471.

\section{*Correspondence to}

Fei Li

Department of Obstetrics

Zhuji Maternal and Child Care Service Center PR China 